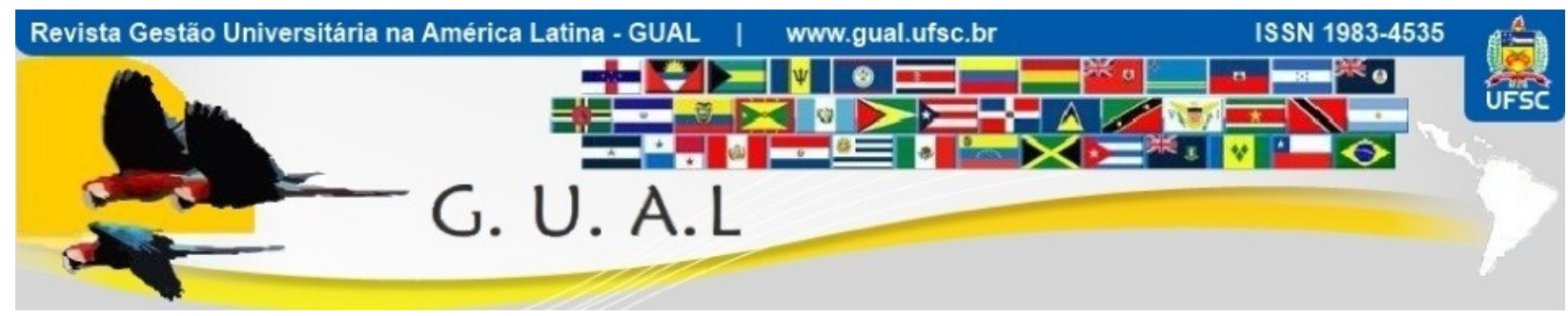

DOI: http://dx.doi.org/10.5007/1983-4535.2015v8n2p50

\title{
BALANCED SCORECARD NA GESTÃO UNIVERSITÁRIA: MAPEAMENTO SOBRE O TEMA UTILIZANDO O PROKNOW-C
}

\section{BALANCED SCORECARD IN HIGHER EDUCATION: MAPPING THE THEME USING THE PROKNOW-C}

Lucas dos Santos Matos, Mestre Universidade Federal de Santa Catarina - UFSC lukxmatos@gmail.com

Sergio Murilo Petri, Doutor Universidade Federal de Santa Catarina - UFSC smpetri@gmail.com

Recebido em 28/fevereiro/2014

Aprovado em 07/abril/2015

Sistema de Avaliação: Double Blind Review

Esta obra está sob uma Licença Creative Commons Atribuição-Uso. 


\title{
RESUMO
}

A crescente importância do ensino superior no cenário econômico e social tem obrigado as universidades a melhorarem seus métodos de gestão, e uma das formas utilizadas tem sido a aplicação do Balanced Scorecard. Esta pesquisa, de natureza exploratório-descritiva, com abordagem quali-quantitativa, objetiva construir o conhecimento necessário sobre a utilização do Balanced Scorecard na gestão universitária, realizando um mapeamento sobre o tema a partir das delimitações dos pesquisadores. Esse conhecimento é tangibilizado pela seleção de artigos relevantes e com reconhecimento científico sobre o tema. É utilizada análise bibliométrica da literatura internacional, por meio do processo Knowledge Development Process - Constructivist (ProKnow-C). Os principais resultados obtidos foram: (i) International Journal of Educational Management é o periódico de maior destaque; (ii) "Commodification of education and academic LABOUR - using the balanced scorecard in a university setting" destaca-se entre os artigos; (iii) Janne Kettunen é o autor mais prolífico.

Palavras-Chave: Balanced Scorecard. Gestão Universitária. Avaliação de desempenho.

\begin{abstract}
The growing importance of higher education in economic and social scenario has forced universities to improve their management methods, and one of the forms used has been the application of the Balanced Scorecard. This research, exploratory and descriptive in nature, with qualitative and quantitative, objective to build the necessary knowledge about the use of the Balanced Scorecard in university management, performing a mapping on the topic from the boundaries of the researchers. This knowledge is made tangible by the selection of relevant articles and with scientific recognition on the topic. It is used bibliometric analysis of international literature, through the process Knowledge Development Process - Constructivist (ProKnow-C). The main results were: (i) International Journal of Educational Management is the period of greatest prominence; (ii) "Commodification of education and academic LABOUR - using the balanced scorecard in a university setting" stands out among the articles; (iii) Janne Kettunen is the most prolific author.
\end{abstract}

Keywords: Balanced Scorecard. University Management. Performance Measurement. 


\section{BALANCED SCORECARD NA GESTÃO UNIVERSITÁRIA: MAPEAMENTO SOBRE O TEMA

\section{INTRODUÇÃO}

A expansão do acesso ao ensino superior e a crescente demanda por qualidade na prestação de serviços nas universidades têm levado estas instituições a aplicarem modelos gerenciais para aperfeiçoarem a sua administração (CULLEN et al., 2003; FRANCESCHINI; TURINA, 2011; CHEN; LEE; MO, 2012). Para aperfeiçoar a gestão universitária pode-se utilizar a avaliação de desempenho, sob o conceito de processo utilizado para construir conhecimento em um decisor, sobre um contexto específico que ele se propõe a gerir (ENSSLIN et al., 2013a). Segundo Nayeri, Mashhadi e Mohajeri (2008), é importante, para a gestão universitária, criar conhecimento sobre a organização para poder aperfeiçoar os seus métodos de gestão.

Um dos mais conhecidos e renomados instrumentos utilizados para mensurar o desempenho empresarial é o Balanced Scorecard (BSC), desenvolvido por Kaplan e Norton (1992). O BSC busca alinhar o planejamento estratégico da empresa com as ações operacionais da empresa, ou seja, alinhar todos objetivos da alta administração, diretoria, gerências e chão de fábrica, buscando uma visão holística de toda a organização (YU; HAMID; IJAB, 2009). O alinhamento é realizado traduzindo, ou materializando-se a estratégia da organização a iniciativas, através de mapas estratégicos (KETTUNEN, 2005; KETTUNEN, 2006).

Vários estudos estão sendo realizados para verificar a aplicação do BSC na gestão universitária, porém ainda de forma muito dispersa, não existindo uma consolidação sobre a utilização do BSC em universidades. Umashankar e Dutta (2007) corrobora, afirmando que há lugares importantes onde não existem esses estudos. Assim, buscando-se preencher esta lacuna existente, visa-se construir um arcabouço teórico, a fim de minimizar retrabalhos em relação a conhecer os principais autores, publicações e periódicos a respeito deste tema específico, com o objetivo de construir conhecimento, acerca deste assunto, nos pesquisadores. O resultado permitirá, também, a outros pesquisadores, uma base de conhecimento sobre como se apresenta, hoje, determinado tema na literatura, apresentando um referencial teórico relevante e reconhecido cientificamente.

Desta forma, para o tema Balanced Scorecard na gestão universitária, esta pesquisa pretende responder aos seguintes questionamentos: (i) Quais os artigos mais relevantes para o tema Balanced Scorecard na gestão universitária? e, (ii) Quais os mais destacados artigos, autores, periódicos e seus respectivos fatores de impacto, e palavras-chave, sobre este tema? 


\section{BALANCED SCORECARD NA GESTÃO UNIVERSITÁRIA: MAPEAMENTO SOBRE O TEMA \\ UTILIZANDO O PROKNOW-C \\ DOI: http://dx.doi.org/10.5007/1983-4535.2015v8n2p50}

Para responder a determinadas perguntas, toma-se como objetivo deste trabalho construir, nos pesquisadores, o conhecimento necessário sobre o tema - aqui ilustrado como Balanced Scorecard na gestão universitária - a partir das delimitações impostas pelos pesquisadores.

A geração deste conhecimento será possível atingindo os seguintes objetivos específicos: (i) selecionar um Portfólio Bibliográfico (PB) relevante, alinhado as delimitações dos pesquisadores, sobre a utilização do BSC na gestão universitária; e, (ii) realizar a análise bibliométrica deste Portfólio Bibliográfico e suas referências, visando identificar os periódicos, artigos, autores e palavras-chave de destaque para o tema de interesse.

Além desta seção introdutória, o artigo apresenta ainda: (i) os métodos de pesquisa; (ii) o instrumento de intervenção utilizado, onde são apresentados os procedimento para formar o PB; (iii) os resultados; e, (iv) as considerações finais. Por fim, são apresentadas as referências utilizadas durante a pesquisa.

\section{MÉTODOS DE PESQUISA}

Esta segunda seção busca apresentar o enquadramento metodológico da pesquisa e ilustrar o processo de pesquisa bibliográfica para a formação do Portfólio Bibliográfico, bem como as delimitações impostas durante a pesquisa. Estas subseções são divididas em (i), (ii) e (iii), respectivamente.

\subsection{ENQUADRAMENTO METODOLÓGICO}

Busca-se definir aqui o enquadramento metodológico desta pesquisa, dividindo-se em seis etapas: i) o objetivo da pesquisa; ii) a lógica da pesquisa; iii) o processo da pesquisa; iv) os procedimentos técnicos; e, v) o instrumento de intervenção utilizado durante a pesquisa.

O enquadramento metodológico, segundo Ensslin et al. (2013a), é visto como a análise, a descrição ou a explicação das abordagens e dos métodos utilizados durante a pesquisa para especificar o conjunto de procedimentos de pressupostos fílosóficos ou disciplinas, os quais fundamentam os temas ou finalidades que explicam ou elucidam o estudo particular para o método científico.

A pesquisa, em relação à natureza de seu objetivo, caracteriza-se como exploratóriodescritiva. É exploratória, pois tem por objetivo construir no pesquisador o conhecimento acerca do tema específico por meio do processo de seleção e análise dos artigos científicos 


\section{BALANCED SCORECARD NA GESTÃO UNIVERSITÁRIA: MAPEAMENTO SOBRE O TEMA \\ UTILIZANDO O PROKNOW-C \\ DOI: http://dx.doi.org/10.5007/1983-4535.2015v8n2p50}

publicados em periódicos, a partir das delimitações atribuídas pelos pesquisadores. É considerada também como descritiva, pois apresenta e descreve as características dos artigos que compõem o Portfólio Bibliográfico por meio de análise bibliométrica, demonstrando, por exemplo, os autores mais prolíficos para o tema (GIL, 1999).

No que se trata da natureza do artigo, a pesquisa é classificada como teóricoilustrativa. A pesquisa busca consolidar informações presentes na literatura internacional sobre a utilização do BSC na gestão universitária, apresentando os passos a serem seguidos durante a pesquisa (ALAVI e CARLSON, 1992).

No que tange a lógica da pesquisa, esta se caracteriza como indutiva, pois parte de uma abordagem genérica para uma abordagem específica, buscando toda uma gama de artigos de um universo limitado para a conclusão de um Portfólio Bibliográfico alinhado e com reconhecimento científico para o tema (ENSSLIN et al., 2013b).

Em relação à coleta de dados, a pesquisa utilizará os dados primários e os dados secundários. Os dados primários podem ser percebidos nas próprias delimitações estabelecidas pelos pesquisadores ao longo do processo de seleção do Portfólio. Os artigos científicos selecionados e analisados caracterizam a utilização de dados secundários durante a pesquisa (RICHARDSON, 1999).

Relativo à abordagem do problema, quanto ao processo de pesquisa, o trabalho é caracterizado, ao mesmo tempo, como qualitativo e quantitativo. É possível verificar a abordagem qualitativa durante o subprocesso de seleção dos artigos que farão parte do Portfólio Bibliográfico para geração de conhecimento, acerca do tema, segundo as percepções dos pesquisadores. Já a abordagem quantitativa fica evidenciada no subprocesso de análise bibliométrica, onde há a contagem das variáveis investigadas, as quais permitirão concluir quais as variáveis que sobressaem às outras (RICHARDSON, 1999).

A classificação em relação aos procedimentos técnicos divide-se em pesquisa bibliográfica e pesquisa-ação. Bibliográfica, pois realizou-se a coleta e análise das publicações científicas, com contribuição de diferentes autores sobre o tema, cuja disponibilização é realizada por meio das bases de dados da Coordenação de Aperfeiçoamento de Pessoal de Nível Superior (CAPES), sendo reconhecidas no domínio científico (GIL, 1999). E pesquisa-ação, pois os pesquisadores estão envolvidos de modo cooperativo e participativo para a resolução do problema de pesquisa, apresentando retro análises de informações durante o processo de seleção do Portfólio Bibliográfico (GIL, 1999). 


\section{BALANCED SCORECARD NA GESTÃO UNIVERSITÁRIA: MAPEAMENTO SOBRE O TEMA \\ UTILIZANDO O PROKNOW-C \\ DOI: http://dx.doi.org/10.5007/1983-4535.2015v8n2p50}

Por último, quanto ao instrumento de intervenção, fora empregado o processo de revisão bibliográfica denominado Knowledge Development Process - Construtivist (ProKnow-C), que, com abordagem construtivista, delineia e apresenta um processo estruturado para construir, no pesquisador, o conhecimento necessário para investigar determinado tema (ENSSLIN et al., 2013a).

\subsection{INSTRUMENTO DE INTERVENÇÃO}

O instrumento de intervenção aplicado a esta pesquisa é o ProKnow-C, cujo objetivo é construir conhecimento, a partir dos interesses e das delimitações impostas pelo pesquisador, segundo a visão construtivista.

O ProKnow-C é composto por quatro etapas: i) seleção de um portfólio bibliográfico sobre o tema; ii) análise bibliométrica do portfólio; iii) análise sistêmica; e, iv) definição da pergunta de pesquisa e objetivo de pesquisa (ENSSLIN et al., 2013b). Para fins desta pesquisa serão aplicadas somente as duas primeiras etapas do ProKnow-C visando o mapeamento sobre o tema, conforme observado da Figura 1.

Quanto ao processo de seleção do Portfólio Bibliográfico, a metodologia consiste em uma série de processos sequenciais que têm início na definição do mecanismo de busca de artigos científicos a ser utilizado, seguido por procedimentos pré-estabelecidos, atingindo a fase de filtragem e seleção de portfólio bibliográfico relevante sobre determinado tema (VALMORBIDA, ENSSLIN e ENSSLIN, 2013). Deve-se salientar que estes artigos correspondem aos que mais se destacaram em termos de reconhecimento científico e alinhamento sobre o tema, segundo a percepção dos pesquisadores.

A etapa seguinte consiste na realização da análise bibliométrica dos artigos do Portfólio Bibliográfico, para verificar quais são os principais periódicos e seus fatores de impacto, artigos, autores e palavras-chave referentes ao tema definido. Realizando-se, assim, o mapeamento do tema (CHAVES et al., 2013). 


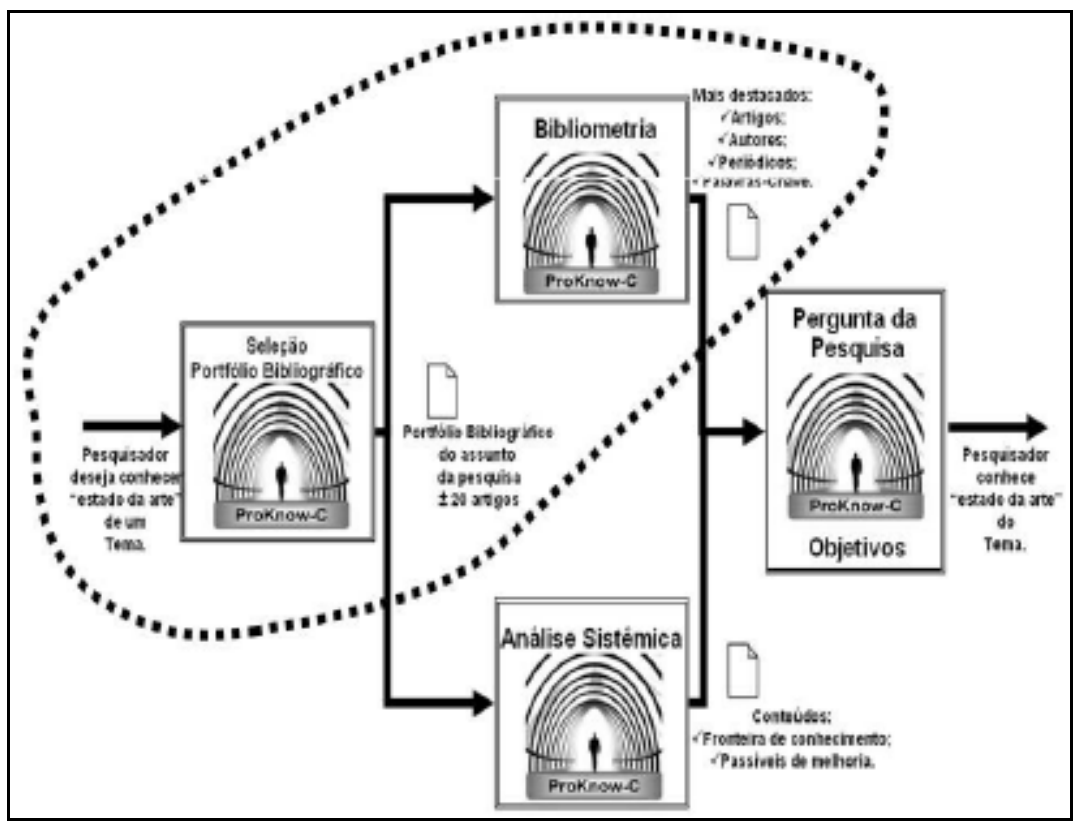

Figura 1 Etapas do ProKnow-C utilizadas na pesquisa Fonte: Lacerda, Ensslin e Ensslin (2012).

\subsection{DELIMITAÇÕES DA PESQUISA}

Para realização da investigação os autores impuseram algumas delimitações. A pesquisa delimitar-se-á: (i) à busca apenas de artigos publicados em periódicos científicos; (ii) à busca de artigos para o PB restritos a artigos internacionais, práticos ou empíricos, publicados a partir do ano 2000; (iii) às bases de dados do Portal de Periódicos da CAPES, com acesso gratuito aos artigos; e (iv) ao alinhamento do entendimento de conteúdo do tema segundo a percepção dos pesquisadores.

\section{PROCESSO DE BUSCA E ANÁLISE DOS ARTIGOS DO PORTFÓlio BIBLIOGRÁFICO}

Para realização da seleção do PB do tema de pesquisa, o processo é constituído de três grandes fases. Primeiramente, dá-se a seleção do banco de artigos brutos; em seguida a filtragem do banco de artigos brutos; e, por fim, o teste de representatividade do PB primário (TASCA et al., 2010).

A primeira grande fase, seleção de artigos brutos, é subdividida em três etapas, que seguem: (i) definição das palavras-chave; (ii) definição dos bancos de dados; (iii) busca dos artigos nos bancos de dados com as palavras-chave; e, (iv) realização de teste de aderência das palavras-chave (ENSSLIN et al., 2013a). 


\section{BALANCED SCORECARD NA GESTÃO UNIVERSITÁRIA: MAPEAMENTO SOBRE O TEMA

A segunda fase, filtragem do banco de artigos, é subdividida em cinco etapas, que são: (i) eliminação de artigos duplicados; (ii) alinhamento pela leitura do título; (iii) alinhamento quanto ao reconhecimento científico; (iv) alinhamento pela leitura do resumo; e (v) alinhamento pela leitura integral dos artigos.

A terceira etapa da seleção do $\mathrm{PB}$, teste de representatividade do $\mathrm{PB}$ primário, é responsável por resgatar possíveis artigos relevantes que por ventura foram descartados em processos anteriores, por meio da análise das referências dos Artigos PB primário.

Esta seleção do portfólio de artigos permite realizar a composição de um Portfólio Bibliográfico, constituído dos artigos considerados mais relevantes na área de conhecimento relativa ao tema de pesquisa, alinhados com a percepção dos pesquisadores, conforme as delimitações estabelecidas por estes para realização da pesquisa.

\subsection{SELEÇÃO DO BANCO DE ARTIGOS BRUTO}

Para se dar início à etapa de seleção do Banco de Artigos Bruto é necessária a definição dos eixos de pesquisa, realizada segundo a percepção dos pesquisadores. O primeiro eixo está intrinsecamente ligado ao tema central do trabalho, ou seja, o Balanced Scorecard. O segundo está relacionado ao local onde se deseja estudar a aplicação da ferramenta, ou seja, na gestão universitária.

Definidos os eixos de pesquisa, passa-se a primeira etapa das quatro definidas anteriormente.

\section{a) Definição das palavras-chave:}

Foram definidas as palavras-chave para os dois eixos de pesquisa descritos anteriormente: Eixo 1 - Balanced Scorecard; e, Eixo 2 - Gestão Universitária. Essa etapa resultou na definição de duas palavras-chave para o primeiro eixo e quatro palavras-chave para o segundo. Resulta então, a partir das combinações das palavras-chave dos eixos de pesquisa, um total de oito combinações de palavras-chave a serem utilizadas na busca por artigos dentro das bases de dados de publicações disponibilizadas pela CAPES. As palavraschave utilizadas no Eixo 1 foram: Balanced Scorecard e BSC. No segundo Eixo, foram definidas as seguintes palavras-chave: Higher Education, Universit*, Undergraduat* $e$ College*. A utilização do asterisco significa um termo "coringa". É utilizado para reunir as 


\section{BALANCED SCORECARD NA GESTÃO UNIVERSITÁRIA: MAPEAMENTO SOBRE O TEMA \\ UTILIZANDO O PROKNOW-C \\ DOI: http://dx.doi.org/10.5007/1983-4535.2015v8n2p50}

variações terminológicas na mesma pesquisa, como por exemplo: Undergraduat* pode significar Undergraduate, Undergraduation e Undergraduates.

\section{b) Definição dos bancos de dados:}

A próxima etapa é a realização da busca pelas bases de dados para a execução da pesquisa. Foram definidos critérios para escolha das bases: (i) constar no Portal de Periódicos da CAPES; (ii) estar contida em uma das duas grandes áreas da CAPES: Ciências Sociais Aplicadas e Engenharias, sendo elas as mais alinhadas com o tema da pesquisa; (iii) disponibilizar ferramenta de busca com o uso de expressões booleanas; (iv) ter ferramentas que disponibilizem a pesquisa nos campos: título (article title), resumo (abstract) e palavraschave (keywords). Outros critérios estabelecidos para as buscas foram a limitação temporal de 13 anos (2000 a 2013) e o tipo de publicação Journal Article. Segundo esses critérios, foram selecionadas 12 bases de dados.

Na sequência, realizou-se o a verificação da representatividade das bases selecionadas, com o teste das combinações de palavras-chave definidas anteriormente. Conforme definição dos pesquisadores, a base seria considerada apta a continuar na pesquisa se retornasse pelo menos um artigo. Atingiu-se o resultado desejado com onze bases de dados: INGENTA CONNECT, ISI WEB OF KNOWLEDGE, HIGHWIRE, SCOPUS, PROQUEST, EMERALD, ENGINEERING VILLAGE, SCIENCEDIRECT, EBSCO, EBSCO UFSC e WILEY. A Base JSTOR não retornou resultados e foi excluída da pesquisa.

\section{c) Busca dos artigos nos bancos de dados com as palavras-chave:}

Essa etapa consistiu em realizar a busca em cada base de dados por meio das combinações de palavras-chave. As oito combinações de palavras-chave pesquisadas nas onze bases de dados resultaram em 1.423 publicações que passaram a compor um portfólio inicial denominado Banco de Artigos Bruto.

\section{d) Realização de teste de aderência das palavras-chave:}

Por fim, para concluir a fase de seleção do banco de artigos bruto para formar o Portfólio Bibliográfico sobre "Balanced Scorecard na gestão universitária", foi realizado o teste de aderência das palavras-chave. O teste foi realizado selecionando-se, aleatoriamente, seis artigos entre as 1.423 publicações que compõem o Banco de Artigos Bruto. Foram 


\section{BALANCED SCORECARD NA GESTÃO UNIVERSITÁRIA: MAPEAMENTO SOBRE O TEMA \\ UTILIZANDO O PROKNOW-C \\ DOI: http://dx.doi.org/10.5007/1983-4535.2015v8n2p50}

identificadas as palavras-chave de cada um dos artigos selecionados, passando-se a verificação da possibilidade destas palavras-chave serem incluídas na pesquisa ou se coincidiam com aquelas definidas para a pesquisa. Como resultado do teste, ficou evidenciado que foram elicitadas 20 palavras-chave pertencentes aos seis artigos, mas nenhuma palavrachave foi adicionada, evidenciando-se ainda a assertividade das palavras-chave definidas. Dessa forma, avançou-se ao desenvolvimento da próxima etapa: filtragem do Banco de Artigos Bruto.

\subsection{FILTRAGEM DO BANCO DE ARTIGOS BRUTO}

$\mathrm{Na}$ etapa de filtragem do Banco de Artigos Bruto, as 1.423 publicações desse Banco foram avaliadas nos seguintes aspectos: i) se os artigos não estão repetidos (redundância); ii) se os títulos dos artigos estão alinhados com o tema da pesquisa; iii) se os artigos possuem reconhecimento científico; iv) se os resumos dos artigos estão alinhados com o tema da pesquisa; e v) se o texto integral dos artigos está alinhado com o tema da pesquisa (LACERDA, ENSSLIN e ENSSLIN, 2012).

Para realização da verificação da redundância dos artigos fora utilizado o software de gerenciamento bibliográfico Endnote (THE THOMSON..., 2008), o qual auxilia na importação das referências encontradas nas bases dados. Depois de realizada a importação, o software permite a exclusão daqueles repetidos. Nesta fase, foram excluídas 603 publicações redundantes, restando 820 publicações não repetidas.

Durante a análise relacionada ao alinhamento do título em relação ao tema da pesquisa, foi detectado que uma quantidade considerável de artigos selecionados estava direcionada a áreas totalmente diferentes à esperada, sendo removidos 737 artigos. Restaram 83 artigos não duplicados e com o título alinhado ao tema da pesquisa.

O próximo aspecto consistiu em verificar o reconhecimento científico dos artigos. Nesse processo, o reconhecimento científico é aferido pelas citações em outros trabalhos científicos. Assim, identificou-se o número de vezes que cada artigo foi citado. Para fins de padronização buscou-se a quantidade de citações de cada artigo no ambiente Google Scholar. Ficou estabelecido pelos autores que seriam considerados os artigos que fossem responsáveis por $90 \%$ de todas as citações dos artigos que passaram pelas fases anteriores. Assim, os artigos que possuem 16 citações, ou mais, foram mantidos no Repositório K (Banco de Artigos não Repetidos e Título Alinhado e com Reconhecimento Científico), que resultou em 


\section{BALANCED SCORECARD NA GESTÃO UNIVERSITÁRIA: MAPEAMENTO SOBRE O TEMA \\ UTILIZANDO O PROKNOW-C \\ DOI: http://dx.doi.org/10.5007/1983-4535.2015v8n2p50}

20 artigos, e os demais foram enviados para o Repositório P (Banco de Artigos não Repetidos e Título Alinhado, com Reconhecimento Científico ainda não confirmado), no total de 63 artigos.

O próximo passo consistiu em realizar a leitura dos resumos dos 20 artigos do Repositório K com o objetivo de verificar o alinhamento do artigo com o tema pesquisado. Nesta etapa, foram considerados alinhados 17 artigos os quais foram armazenados no Repositório A (Banco de Artigos não Repetidos e Título e Resumo Alinhado e com Reconhecimento Científico). Os demais, três artigos, foram eliminados.

A etapa seguinte teve o objetivo de filtrar aqueles artigos que haviam reconhecimento científico não confirmado, ou seja, os artigos do Repositório P. Os 63 artigos com menor reconhecimento científico foram analisados para identificar quais continuariam no processo. Nesta etapa são considerados fatores como período de publicação e se os autores possuem reconhecimento científico, estando presentes como autores dos artigos do Repositório A. Esta etapa selecionou 3 artigos potenciais, os quais foram depositados no Repositório B.

Na sequência, mesclou-se os artigos armazenados nos Repositórios A (17 artigos) e B (3 artigos) formando um único, denominado Repositório C, composto por 20 artigos.

Finalizando a etapa de filtragem do Banco de Artigos Bruto, os 20 artigos constantes no Repositório C foram analisados em relação ao alinhamento do texto integral com o tema da pesquisa. Primeiramente é verificado se o artigo está disponível gratuitamente, via internet ou via Portal de Periódicos da CAPES. Apenas um dos artigos não estava disponível, restando 19 artigos, os quais passaram a verificação do alinhamento integral do artigo. Esta fase terminou com a seleção de 14 artigos considerados integralmente alinhados com o tema da pesquisa, os quais passam a integrar o bando denominado Artigos Primários do Portfólio Bibliográfico.

\subsection{TESTE DE REPRESENTATIVIDADE DO PB}

A partir dos Artigos Primários do Portfólio Bibliográfico foram buscadas suas referências bibliográficas, objetivando obter a relação de todos os artigos científicos ali constantes. Primeiramente foram constatadas 413 referências brutas. Após o tratamento, eliminação de referências duplicadas e eliminação de referências que não fossem do tipo Jounal Article, desalinhadas ao tema de pesquisa e publicadas antes de 2000, têm-se como resultado 17 artigos. Destes artigos, fora realizada uma análise representando $80 \%$ das 


\section{BALANCED SCORECARD NA GESTÃO UNIVERSITÁRIA: MAPEAMENTO SOBRE O TEMA \\ UTILIZANDO O PROKNOW-C \\ DOI: http://dx.doi.org/10.5007/1983-4535.2015v8n2p50}

citações totais, determinado pelo Google Scholar, buscando incluir artigos alinhados com representação científica que por algum motivo tenham sido incluídas neste Portfólio Primário.

Esses $80 \%$ foram representados por 7 artigos, com ponto de corte em 59 citações. Dos 7 artigos restantes, 4 deles já estavam contidos nos Artigos Primários do Portfólio Bibliográfico, restando 3 artigos para re-análise. Esse três artigos são analisados nos aspectos do alinhamento do resumo e integralidade com o tema de pesquisa. Dois artigos considerados alinhados em relação ao seu resumo continuam para etapa de verificação do alinhamento integral do artigo. Esses dois artigos não puderam ser acessados na íntegra, e são eliminados da pesquisa.

O processo de filtragem resultou em um Portfólio Bibliográfico formado pelos 14 artigos, listados no Quadro 1.

\section{Portfólio Bibliográfico}

1. LAWRENCE, S. e SHARMA, U. Commodification of education and academic LABOUR - using the balanced scorecard in a university setting. Critical perspectives on accounting, v. 13, n. 5, p. 661-677, 2002.

2. CULLEN, J. et al. Quality in higher education: from monitoring to management. Quality Assurance in Education, v. 11, n. 1, p. 5-14, 2003.

3. KETTUNEN, J. Implementation of strategies in continuing education. International Journal of Educational Management, v. 19, n. 3, p. 207-217, 2005.

4. KETTUNEN, J. Strategic planning of regional development in higher education. Baltic Journal of Management, v. 1, n. 3, p. 259-269, 2006.

5. PAPENHAUSEN, C. e EINSTEIN, W. Implementing the Balanced Scorecard at a college of business. Measuring Business Excellence, v. 10, n. 3, p. 15-22, 2006.

6. CHEN, S. et al. The application of balanced scorecard in the performance evaluation of higher education. The TQM Magazine, v. 18, n. 2, p. 190-205, 2006.

7. NAYERI, M. D. et al. Universities strategic evaluation using balanced scorecard. World Academy of Science, Engineering and Technology, v. 37, p. 332-337, 2008.

8. UMASHANKAR, V. e DUTTA, K. Balanced scorecards in managing higher education institutions: an Indian perspective. International Journal of Educational Management, v. 21, n. 1, p. 54-67, 2007.

9. MCDEVITT, R. et al. Strategy revitalization in academe: a balanced scorecard approach. International Journal of Educational Management, v. 22, n. 1, p. 32-47, 2008.

10. YU, M. L. et al. The e-balanced scorecard (e-BSC) for measuring academic staff performance excellence. Higher Education, v. 57, n. 6, p. 813-828, 2009.

11. WU, H. et al. Performance evaluation of extension education centers in universities based on the balanced scorecard. Evaluation and Program Planning, v. 34, n. 1, p. 37-50, 2011.

12. SCHOBEL, K. e SCHOLEY, C. Balanced Scorecards in education: focusing on financial strategies. Measuring Business Excellence, v. 16, n. 3, p. 17-28, 2012.

13. CHEN, Y. et al. Innovative Operation In A Private University Of Technology--An Application Of Strategy Map On Balanced Scorecard. International Journal of Asian Social Science, v. 2, n. 11, p. 1877-1891, 2012.

14. FRANCESCHINI, F. e TURINA, E. Quality improvement and redesign of performance measurement systems: an application to the academic field. Quality \& Quantity, v. 47, n. 1, p. 465-483, 2013.

Quadro 1 Portfólio final de artigos

Fonte: Dados da pesquisa. 


\subsection{ANÁLISE BIBLIOMÉTRICA}

Finalizada a seleção do Portfólio Bibliográfico, seguindo a metodologia ProKnow-C, passa-se à etapa de Análise Bibliométrica dos artigos do PB. Essa bibliometria consiste na aplicação de procedimentos matemáticos e estatísticos de um conjunto definido de artigos - no caso o Portfólio Bibliográfico - para a gestão da informação e do conhecimento científico do tema de pesquisa (TASCA et al., 2010; ENSSLIN et al., 2013b).

A realização da análise bibliométrica do Portfólio Bibliográfico estuda quatro aspectos: i) grau de relevância dos periódicos e respectivos fatores de impacto; ii) reconhecimento científico dos artigos; iii) grau de relevância dos autores; e, iv) palavraschave mais utilizadas.

Cada um dos aspectos, com exceção da estimativa de palavras-chave mais utilizadas e da estimativa do fator de impacto dos periódicos do Portfólio Bibliográfico, terá a realização das análises na seguinte ordem: i) análise bibliométrica dos artigos do Portfólio Bibliográfico; ii) análise bibliométrica das referências dos artigos do Portfólio Bibliográfico; e iii) análise bibliométrica do conjunto formado pelos artigos e suas próprias referências.

$\mathrm{O}$ conjunto de dados a serem submetidos à análise bibliométrica são os 14 artigos pertencentes ao Portfólio Bibliográfico e suas respectivas 200 referências bibliográficas. É importante salientar que foram consideradas apenas as referências do tipo journal article a partir do ano 2000.

\subsubsection{Grau de relevância dos periódicos}

Esta primeira análise tem por objetivo identificar em quais periódicos foi publicado o maior número de artigos dentre os que compõem o PB. O resultado evidenciou que os artigos foram publicados em 11 periódicos diferentes, observando-se que o periódico International Journal of Educational Management é o que apresenta maior grau de relevância com três artigos publicados, dentre os do PB.

A segunda análise objetiva identificar em quais periódicos foi publicado o maior número de artigos dentre os citados nas referências do PB. O periódico International Journal of Educational Management se mostra novamente como relevante, contendo 14 dos artigos das Referências do PB. A Harvard Business Review e a International Journal of Operations \& Production Management também apresentam grande relevância, com 11 e 10 artigos, respectivamente. 
A terceira análise realiza uma comparação entre a relevância dos periódicos dos artigos do PB e a relevância dos periódicos das referências deste Portfólio. Confirmando as informações anteriores, o periódico International Journal of Educational Management é considerado como de destaque, tanto no $\mathrm{PB}$, quanto em suas referências. O resultado é apresentado no Gráfico 1.

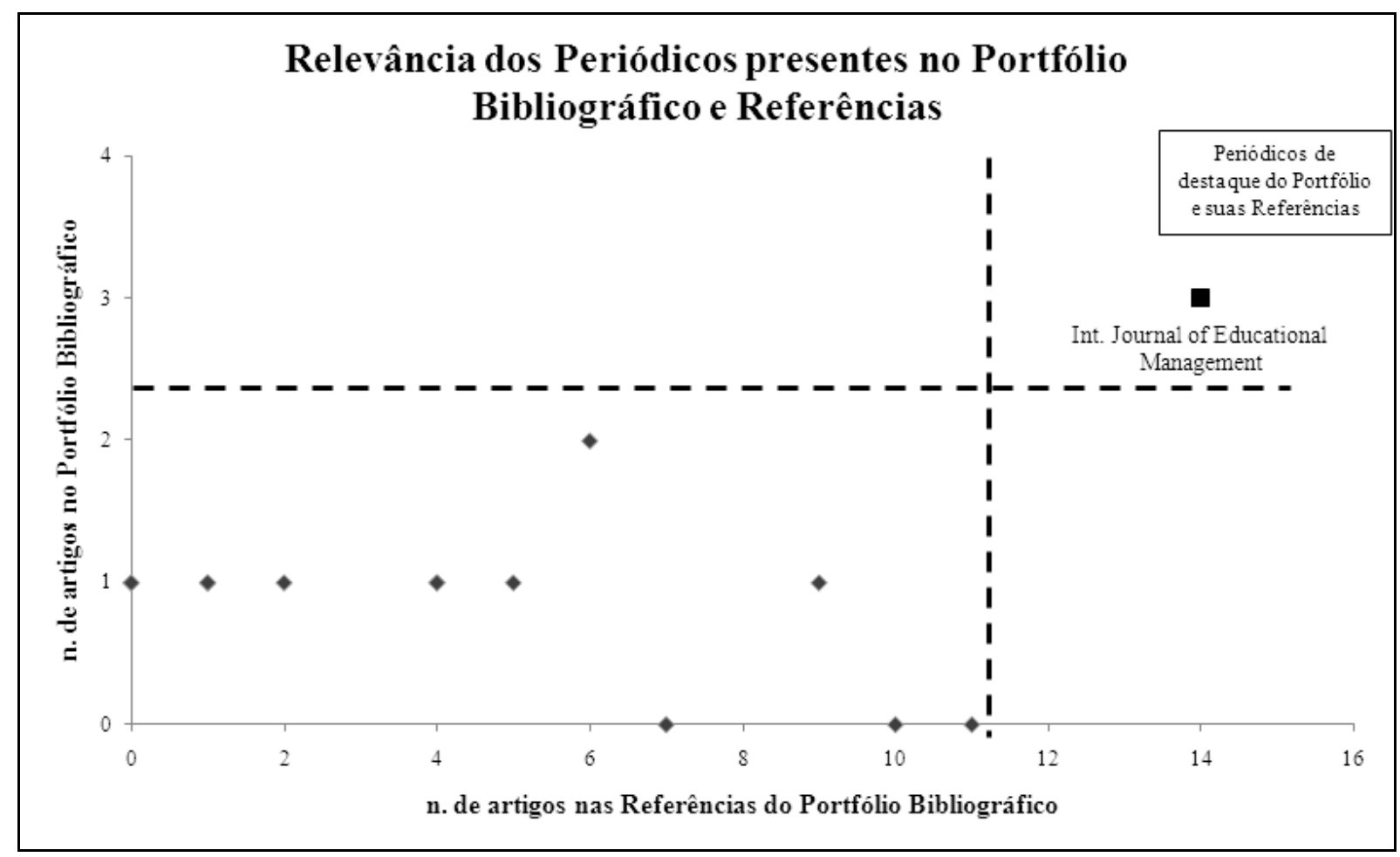

Gráfico 1 Relevância dos periódicos presentes nos artigos e nas referências da pesquisa Fonte: Dados da pesquisa.

A evidenciação do periódico mais relevante para o tema apresenta aos pesquisadores possíveis alvos para publicação de materiais referentes a este tema e potencial fonte de dados para futuras pesquisas.

Em relação aos fatores de impacto dos periódicos, o periódico Higher Education foi o que apresentou melhor relação entre JCR e SJR, registrando 0,937 e 1,112, respectivamente, daqueles presentes no PB. O periódico mais presente na pesquisa, International Journal of Educational Management apresenta somente o SJR, de 0,391.

\subsubsection{Grau de reconhecimento científico dos artigos}

O reconhecimento científico é mensurado, para fins desta pesquisa, segundo o número de citações encontrado para o artigo, utilizando a base do Google Scholar. 
A primeira análise realizada busca estimar o reconhecimento científico dos artigos do PB, destacando-se como de maior relevância o artigo de Lawrence e Sharma (2002). Esse artigo foi citado 152 vezes até a data da presente pesquisa (dezembro de 2013), o que reflete o reconhecimento científico do artigo para o tema pesquisado.

A segunda análise tem por objetivo avaliar o reconhecimento científico dos artigos do PB nas referências presentes nos artigos do Portfólio. Neste aspecto, o artigo Umashankar e Dutta (2007) se mostrou bastante relevante, pois foi citado 5 vezes por outros artigos do PB.

Realiza-se a terceira análise comparando-se o número de citações do artigo do Portfólio Bibliográfico e o número de citações obtidas pelo autor mais citado de cada um dos artigos nas referências desse Portfólio, com base no Google Scholar. Como resultado obtevese: i) nenhum artigo de destaque do PB foi realizado por autor de destaque das referências do PB; ii) o artigo de Lawrence e Sharma (2002) destaca-se no PB; e, iii) Os artigos Kettunen (2005) e Kettunen (2006) foram realizados por autor de destaque nas referências do PB, sendo o autor responsável por 6 artigos das Referências do PB. Os resultados obtidos com essa análise estão apresentados no Gráfico 2.

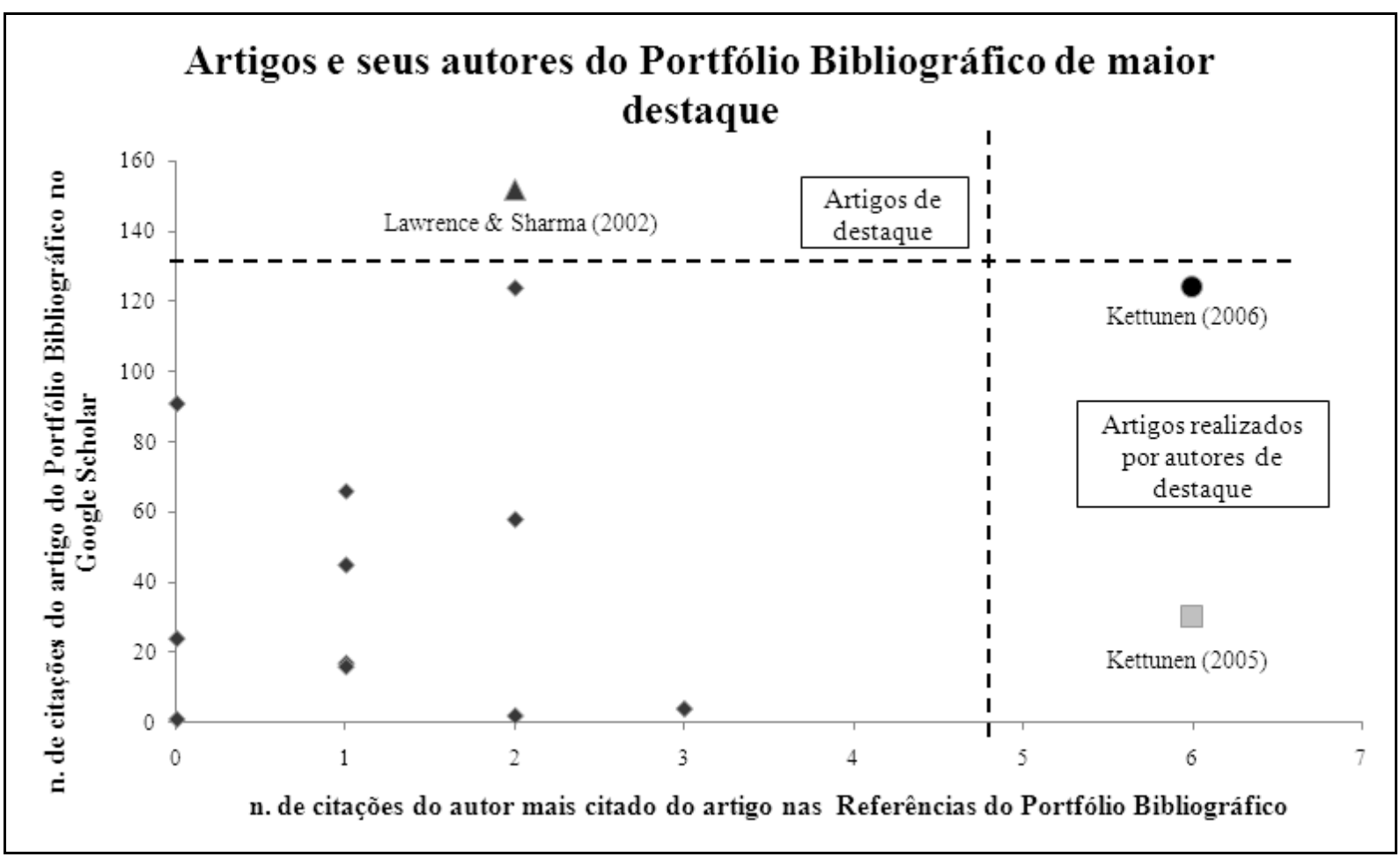

Gráfico 2 Artigos e seus autores do Portfólio Bibliográfico de maior destaque Fonte: Dados da pesquisa. 
Os resultados a respeito dos artigos mais relevantes para o tema, segundo as percepções dos pesquisadores, evidenciam o reconhecimento científico e a importância dessas publicações para a realização de pesquisas alinhadas a este tema. Artigos bem citados demonstram sua aceitação no meio científico e trazem consigo conceitos utilizados para discussões acerca do tema, considerando-os artigos seminais.

\subsubsection{Grau de relevância dos autores}

O grau de relevância dos autores se inicia com a análise da importância dos autores do PB. Dentre os 34 autores dos artigos do PB, foi considerado mais relevante o Professor Janne Kettunen, autor de dois artigos do PB.

A segunda análise busca evidenciar quais os autores de maior relevância entre os autores das referências dos artigos do PB. Como esperado, devido a forte ligação da ferramenta Balanced Scorecard com seus autores, ficou evidenciada a grande participação de Robert Kaplan e David Norton, com 12 e 10 artigos, respectivamente, nas referências do PB.

A terceira análise realizada comparou o número de artigos realizados pelos autores no PB e suas referências, evidenciando-se que: i) nenhum autor foi considerado de destaque no PB e suas referências; ii) os autores de maior destaque nas referências do PB são Kaplan e Norton; e, iii) o autor de maior destaque nos artigos do PB é o Professor Janne Kettunen. Os resultados dessa análise estão demonstrados no Gráfico 3.

A evidenciação acerca dos autores mais prolíficos permite conhecer quem são os maiores pesquisadores sobre o tema, segundo a percepção dos pesquisadores deste artigo, e verificar características a respeito desses autores, como quais são as linhas de pesquisa que estudam, suas experiências profissionais, quais são os trabalhos que estão realizando e quais são as evoluções e perspectivas sobre o tema (ENSSLIN et al., 2013b). 


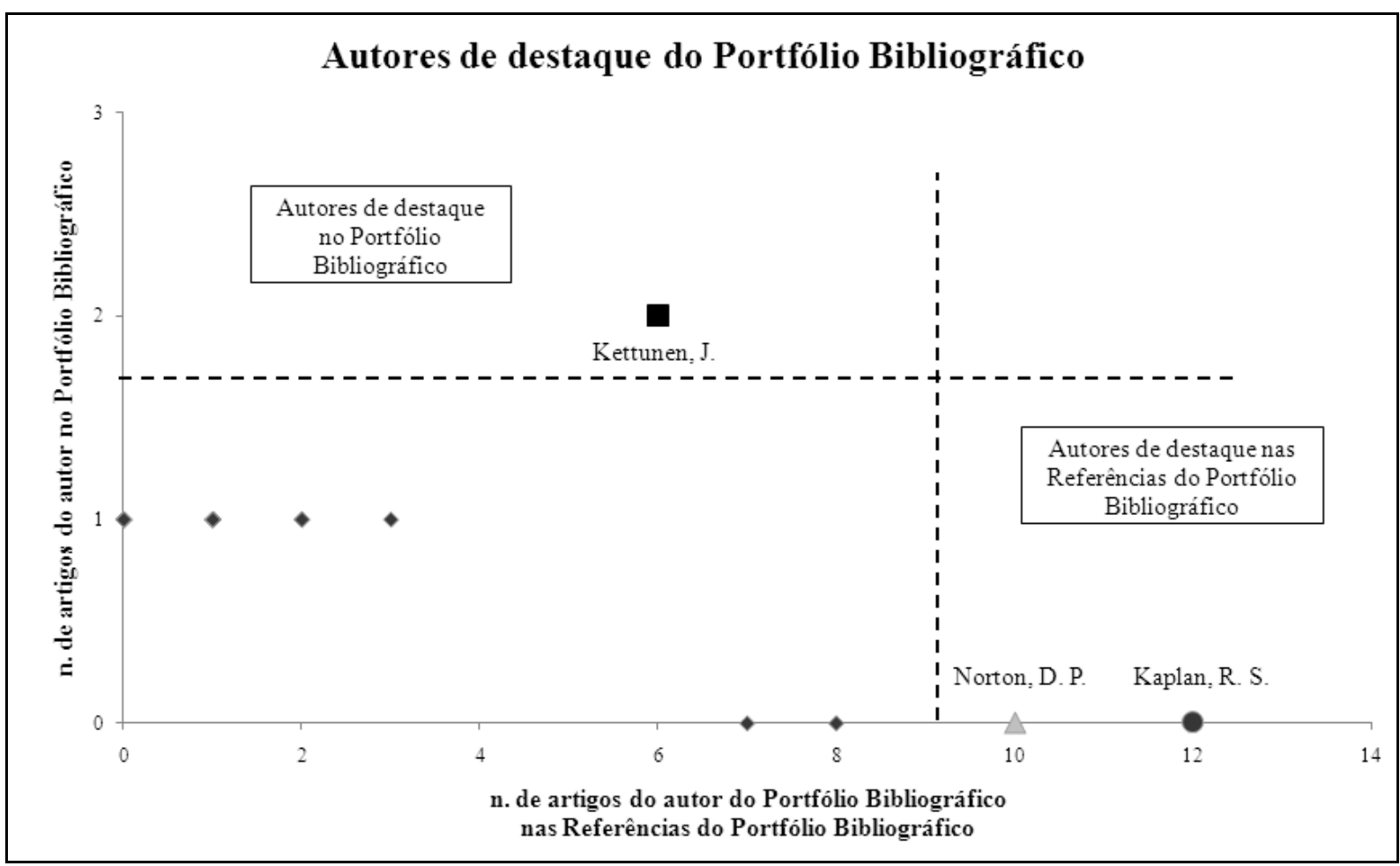

Gráfico 3 Autores de destaque do Portfólio Bibliográfico Fonte: Dados da pesquisa.

\subsubsection{Palavras-chave mais utilizadas}

A última análise da bibliometria busca identificar quais são as palavras-chave mais utilizadas nos artigos do Portfólio Bibliográfico. A evidenciação destas palavras-chave permite auxiliar futuras pesquisas relacionadas ao tema, e também como teste de aderência das palavras-chave definidas no início da seleção de artigos nas bases de dados.

O resultado desta etapa evidenciou que as duas palavras-chave mais presentes já haviam sido utilizadas no início da pesquisa. São elas Balanced Scorecard, presente em 13 artigos do PB, e Higher Education, presente em 10 artigos do PB. Nenhuma outra palavrachave foi considerada relevante para a realização da pesquisa.

\section{CONSIDERAÇÕES FINAIS}

A crescente importância do ensino superior no cenário econômico e social tem levado as universidades a tentarem melhor sua gestão. Para isso, é necessário utilizar ferramentas que as auxiliem nesse processo. Uma das principais ferramentas utilizadas é a de avaliação de desempenho, desenvolvida por Kaplan e Norton (1992), denominada Balanced Scorecard. 


\section{BALANCED SCORECARD NA GESTÃO UNIVERSITÁRIA: MAPEAMENTO SOBRE O TEMA

Este estudo buscou realizar um mapeamento sobre este tema, aplicando o processo de construção de conhecimento ProKnow-C (TASCA et al., 2010; LACERDA, ENSSLIN e ENSSLIN, 2012), objetivando identificar, na literatura internacional qualificada, quais os principais artigos publicados sobre o tema com reconhecimento científico; quem são os pesquisadores que estão tratando do tema; quais os periódicos mais relevantes e seus respectivos fatores de impacto; e quais as palavras-chave mais utilizadas. Assim, alcançar-seia o objetivo deste artigo e responder-se-ia as questões de pesquisa.

A partir dos 14 artigos selecionados utilizando-se o ProKnow-C, os quais compõem o Portfólio Bibliográfico, analisou-se alguns aspectos pontuais. Os principais resultados do perfil das publicações relevantes sobre o tema são: (i) o periódico International Journal of Educational Management é o mais relevante para o PB e suas referências; (ii) Lawrence e Sharma (2002) é o artigo mais relevante do PB e os artigos Kettunen (2005) e Kettunen (2006) são realizados por autores de destaque nas referências do PB; (iii) Janne Kettunen é o autor mais relevante para o PB e Robert Kaplan e David Norton são os autores mais presentes na referências do PB; e, (iv) as palavras-chave mais presentes no PB são Balanced Scorecard

\section{e Higher Education.}

A contribuição deste trabalho se sustenta no resgate do conhecimento existente e que está disseminado em vários veículos de comunicação ao apresentar e ilustrar um processo de seleção de artigos relevantes que irão compor o referencial teórico de uma pesquisa que investigue a utilização do Balanced Scorecard na gestão universitária.

Após a construção de todo esse conhecimento, pode-se propor a continuação desta pesquisa, com a realização da análise do conteúdo, ou análise sistêmica, dos artigos selecionados com vistas a identificar oportunidades de pesquisa sobre o tema, podendo passar à terceira e quarta etapa do ProKnow-C.

\section{REFERÊNCIAS}

ALAVI, M.; CARLSON, P. A review of MIS research and disciplinary development. Journal of Management Information Systems, v. 8, n. 4, p. 45-62, 1992.

CHAVES, L. C.; FREITAS, C. L.; ENSSLIN, L.; PFITSCHER, E. D.; PETRI, S. M.; ENSSLIN, S. R. Gestão ambiental e sustentabilidade em instituições de ensino superior: construção de conhecimento sobre o tema. Revista Gestão Universitária na América Latina, v. 6, n. 2 , p. 33-54, 2013. 


\section{BALANCED SCORECARD NA GESTÃO UNIVERSITÁRIA: MAPEAMENTO SOBRE O TEMA \\ UTILIZANDO O PROKNOW-C \\ DOI: http://dx.doi.org/10.5007/1983-4535.2015v8n2p50}

CHEN, S.; YANG, C.; SHIAU, J. The application of balanced scorecard in the performance evaluation of higher education. The TQM Magazine, v. 18, n. 2, p. 190-205, 2006.

CHEN, Y.; LEE, S.; MO, H. Innovative Operation In A Private University Of Technology-An Application Of Strategy Map On Balanced Scorecard. International Journal of Asian Social Science, v. 2, n. 11, p. 1877-1891, 2012.

CULLEN, J.; JOYCE, T.; HASSALL, T.; BROADBENT, M. Quality in higher education: from monitoring to management. Quality Assurance in Education, v. 11, n. 1, p. 5-14, 2003. ENSSLIN, S. R.; ENSSLIN, L.; LACERDA, R. T. O.; MATOS, L. S. Evidenciação do estado da arte do tema avaliação do desempenho na regulação de serviços públicos segundo a percepção dos pesquisadores. Gestão Pública: Práticas e Desafios, v. 4, p. 121-147, 2013a.

ENSSLIN, S. R.; ENSSLIN, L.; MATOS, L. S.; RIPOLL-FELIU, V. M. Uma análise dos destaques e oportunidades sobre o tema 'Avaliação de desempenho na regulação dos serviços públicos'. In: Congresso Internacional de Contabilidade e Auditoria, 14, 2013, Lisboa Anais..., Lisboa, $2013 b$.

FRANCESCHINI, F.; TURINA, E. Quality improvement and redesign of performance measurement systems: an application to the academic field. Quality \& Quantity, v. 47, n. 1, p. 465-483, 2013.

GIL. A. C. Métodos e técnicas de pesquisa social. São Paulo: Atlas, 1999.

KAPLAN, R.S.; NORTON, D. P. The balanced scorecard measures that drive performance. Harvard Business Review, v. 70, n. 1, p. 71-79, 1992.

KETTUNEN, J. Implementation of strategies in continuing education. International Journal of Educational Management, v. 19, n. 3, p. 207-217, 2005.

Strategic planning of regional development in higher education. Baltic Journal of Management, v. 1, n. 3, p. 259-269, 2006.

LACERDA, R. T. O.; ENSSLIN, L.; ENSSLIN, S. R. Uma análise bibliométrica da literatura sobre estratégia e avaliação de desempenho. Gestão \& Produção, v. 19, n. 1, p. 59-78, 2012.

LAWRENCE, S.; SHARMA, U. Commodification of education and academic LABOUR using the balanced scorecard in a university setting. Critical perspectives on accounting, v. 13, n. 5, p. 661-677, 2002.

MCDEVITT, R.; GIAPPONI, C.; SOLOMON, N. Strategy revitalization in academe: a balanced scorecard approach. International Journal of Educational Management, v. 22, n. 1, p. 32-47, 2008.

NAYERI, M. D.; MASHHADI, M. M.; MOHAJERI, K. Universities strategic evaluation using balanced scorecard. World Academy of Science, Engineering and Technology, v. 37, p. 332-337, 2008. 
PAPENHAUSEN, C.; EINSTEIN, W. Implementing the Balanced Scorecard at a college of business. Measuring Business Excellence, v. 10, n. 3, p. 15-22, 2006.

RICHARDSON, R. J. Pesquisa social: métodos e técnicas. São Paulo: Atlas, 1999.

SCHOBEL, K.; SCHOLEY, C. Balanced Scorecards in education: focusing on financial strategies. Measuring Business Excellence, v. 16, n. 3, p. 17-28, 2012.

TASCA, J. E.; ENSSLIN, L.; ENSSLIN, S. R.; ALVES; M. B. M. An approach for selecting a theoretical framework for the evaluation of training programs. Journal of European Industrial Training, Vol. 34, N. 7, p. 631-655, 2010.

THE THOMSON CORPORATION. EndNote X. 2. ed. The Thomson Corporation, 2008.

UMASHANKAR, V.; DUTTA, K. Balanced scorecards in managing higher education institutions: an Indian perspective. International Journal of Educational Management, v. 21, n. 1, p. 54-67, 2007.

VALMORBIDA, S. M. I.; ENSSLIN, S. R.; ENSSLIN, L. Avaliação de Desempenho na Administração de Universidade Pública: Análise Bibliométrica da Literatura Nacional e Internacional. Administração Pública e Gestão Social, v. 5, p. 116-125, 2013.

WU, H.; LIN, Y.; CHANG, C. Performance evaluation of extension education centers in universities based on the balanced scorecard. Evaluation and Program Planning, v. 34, n. 1, p. 37-50, 2011.

YU, M. L.; HAMID, S.; IJAB, M. T. The e-balanced scorecard (e-BSC) for measuring academic staff performance excellence. Higher Education, v. 57, n. 6, p. 813-828, 2009. 